\title{
Sु \\ Controlled sequential dehydrogenation of single molecules by scanning tunneling microscopy
}

\author{
Nadjib Baadji, ${ }^{1}$ Stefan Kuck, ${ }^{2}$ Jens Brede, ${ }^{2}$ Germar Hoffmann, ${ }^{2}$ Roland Wiesendanger, ${ }^{2}$ and Stefano Sanvito ${ }^{1}$ \\ ${ }^{1}$ School of Physics and CRANN, Trinity College, Dublin 2, Ireland \\ ${ }^{2}$ Institut für Angewandte Physik, Universität Hamburg, Hamburg, Germany
}

(Received 30 June 2010; published 27 September 2010)

\begin{abstract}
Scanning tunneling microscopy (STM) is today the most powerful and versatile tool available for imaging and manipulating single molecules on surfaces. Here, we explore its ultimate limit by demonstrating the possibility of controlling sequential di-dehydrogenation of single Co-Salen molecules sublimated on $\mathrm{Cu}$. In particular, we are able to explore the final products of the $\mathrm{H}_{2}$ dissociation as well as the intermediate state, in which only one $\mathrm{H}$ atom is separated from the molecule. This is achieved by low-temperature STM with the dissociation induced by either point spectroscopy or in the standard constant-current mode. Crucial for the interpretation of the data is our ability to perform state-of-the-art density-functional theory simulations of both topographic and spectroscopical STM images. This work demonstrates that STM combined with theory can give access to the atomic details of a chemical reaction even when the reaction products are completely unknown.
\end{abstract}

DOI: 10.1103/PhysRevB.82.115447

PACS number(s): 68.37.Ef, 68.43.Fg, 61.46.-w, 71.15.Mb

\section{INTRODUCTION}

The practice of inducing chemical reactions by passing a current through molecules deposited on surfaces is today well established in the scanning tunneling microscopy (STM) community. The basic concept includes selectively exciting those particular vibrational modes driving the desired reaction. This strategy has been adopted to move, desorb, or dissociate small molecules on surfaces, ${ }^{1-4}$ to fuse two molecules together, ${ }^{5}$ or to manipulate the bonding geometry between the molecule and the surface. ${ }^{6,7}$ In most of these situations it is easy to establish that a reaction has occurred from a clear change in the topographic STM images before and after the reaction. For instance, the fingerprint of a dissociation is the detection of isolated byproducts around the position of the original molecule. ${ }^{3}$ In some cases one can associate different molecules to seemingly similar topographic images, differing only by their orientation with respect to a known crystallographic direction of the substrate. ${ }^{6}$ The identification of the reaction products then proceeds by chemical intuition and by comparing the topographic images with those of known molecules. Sometime this is helped by a further comparison between the inelastic electron tunneling spectrum with available data on vibrational modes of the molecule. ${ }^{8}$

A more complex situation is given when the system appears little "talkative," i.e., when the chemical reaction does not produce a major change in the geometry. An extreme case is represented by dehydrogenation of planar molecules. Here, one of the products of the reaction, the hydrogen, leaves the surface and therefore is not detectable. The other one, the molecule, changes only little its geometry. The study of such a reaction poses the ultimate challenge for STM.

The molecules of choice for this work are Co- $\mathrm{N}, \mathrm{N}^{\prime}$-ethylenebis (salicydeneiminato) complexes (hereafter "Co-Salen"). Salens are versatile objects, ${ }^{9}$ which allow for the incorporation of a range of transition metals so that their magnetic properties can be finely tuned. Due to their given planarity and robustness, Salens are also ideally suited to locally probe chemical and magnetic properties by scanning probe techniques. ${ }^{10,11}$ In Co-Salen, the Co ion is bonded to two $\mathrm{O}$ and two $\mathrm{N}$ atoms. These form with the ethylene bridge $\left(\mathrm{C}_{2} \mathrm{H}_{4}\right)$ a di-aminoethane $\left(\mathrm{C}_{2} \mathrm{H}_{4} \mathrm{~N}_{2}\right)$ group (see Fig. 1) from which the hydrogen will be sequentially extracted in a STM-controlled dehydrogenation process.

In this work we will demonstrate the ability of STM to access not only the final products of the $\mathrm{H}_{2}$ dissociation but also the intermediate state in which only one $\mathrm{H}$ atom is separated from the molecule. We will first introduce both our experimental and theoretical methods and then we will demonstrate the controlled dissociation. During the discussion STM experimental data will be extensively compared with the simulated images and the quantitative agreement between the two will form the basis for our argument. Finally we will draw some conclusions.

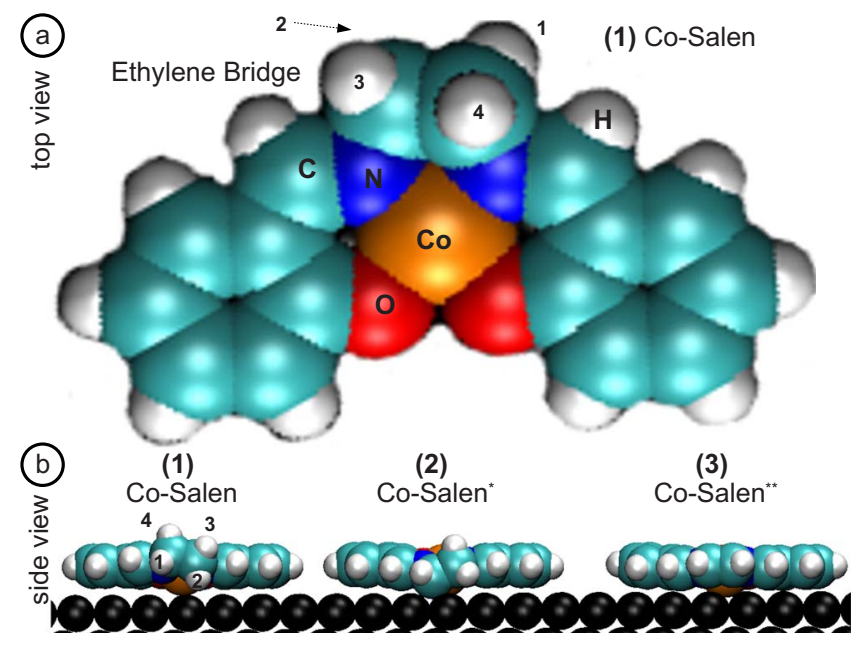

FIG. 1. (Color online) The three Co-Salen molecules investigated in this work: (1) intact $\left(\mathrm{C}_{2} \mathrm{H}_{4} \mathrm{~N}_{2}\right.$ apex), (2) single dehydrogenated $\left(\mathrm{C}_{2} \mathrm{H}_{3} \mathrm{~N}_{2}\right.$ apex), and (3) double dehydrogenated $\left(\mathrm{C}_{2} \mathrm{H}_{2} \mathrm{~N}_{2}\right.$ apex). Top and side views show the structures after numerical relaxation on the $\mathrm{Cu}(111)$ surface. 


\section{METHOD}

\section{A. Sample preparation and STM measurements}

Tips and $\mathrm{Cu}(111)$ surfaces are prepared by standard procedures (alternating cycles of argon-ion etching and annealing) in an ultrahigh-vacuum environment. In situ, Co-Salen molecules are dosed onto room-temperature $\mathrm{Cu}(111)$ surfaces by sublimation from homebuilt Knudsen cells. Thereby, molecules are highly mobile and $\mathrm{Cu}(111)$ step edges are completely decorated. Due to repulsive interaction among adjacent molecules Co-Salens stay isolated. These are subject of the present investigation. Then after the temperature is decreased, surfaces are investigated at $25 \mathrm{~K}$ in a variable-temperature STM, ${ }^{12}$ and spectroscopic results are reconfirmed with higher energy resolution in a bath cryostat system operated at $7 \mathrm{~K} .^{13}$

STM images presented are obtained in the constantcurrent mode at the indicated current settings and bias voltages. Voltages refer to the potential of the tip relative to the sample, with positive voltages associated to tunneling into unoccupied sample states and negative voltages to tunneling out of the occupied ones. The feedback signal, i.e., the tipsample distance in the constant-current mode is recorded and plotted color encoded.

For the acquisition of the differential conductivity, a modulation voltage $(\Delta U=5 \mathrm{mV}, f=2.77 \mathrm{kHz})$ is added to the carrier voltage and the modulation of the current due to contributions of the local density of states at the appropriate energy is recorded by a lock-in amplifier (SR-830). Thereby, maps of the differential conductivity are obtained in parallel to constant-current images, i.e., with the feedback loop being active. Scanning tunneling spectroscopy (STS) data are obtained at selected positions, i.e., the feedback is disabled, the $(x, y, z)$ coordinates of the tip are kept fixed and the carrier voltage is ramped.

\section{B. Simulated STM images}

Simulated data are obtained from density-functional theory (DFT) combined with the perturbative Bardeen's approach $^{14}$ for evaluating the STM current. DFT calculations are performed by using the localized pseudoatomic-orbital basis and pseudopotential code SIESTA. ${ }^{15}$ An optimized double- $\zeta$ basis set is defined in the split valence manner, where only the valence electrons are treated explicitly. These are, respectively, $1 s^{1}, 2 s^{2} 2 p^{2}, 2 s^{2} 2 p^{3}, 2 s^{2} 2 p^{4}, 4 s^{2} 4 p^{0} 3 d^{7}$, and $4 s^{2} 4 p^{0} 3 d^{10}$ for $\mathrm{H}, \mathrm{C}, \mathrm{N}, \mathrm{O}, \mathrm{Co}$, and $\mathrm{Cu}$. The core electrons for each atomic specie are described by norm-conserving Troullier-Martin's pseudopotentials ${ }^{16}$ with nonlinear core correction. ${ }^{17}$ The generalized gradient approximation with the standard Perdew-Burke-Ernzerhof (PBE) (Ref. 18) parametrization is used to describe the exchange-correlation potential. The atomic relaxation of the system is achieved up to a maximum force below $0.05 \mathrm{eV} / \AA$. The obtained relaxed structure for $\mathrm{Co}-\mathrm{Salen}$ is in good agreement with previous calculation. ${ }^{10}$

The STM images, both in the topographic and spectroscopical modes, are calculated with a modified version of the Tersoff-Hamann scheme, ${ }^{19}$ which takes into account the electronic structure of the tip. In practice we separate the computation of the STM images into two independent calculations for the electronic structure of the sample (molecule plus substrate) and the STM tip. These provide the electron density over a real-space grid in the proximity of the sample and of the tip (approximately up to $3 \AA$ ) but not in the vacuum region. We then propagate the electron density of the sample in the vacuum by analytic continuation with onedimensional decaying plane waves. Note that an alternative way of propagating the wave function in the vacuum is that of using Hankel functions, i.e., solutions of the Helmotz equation with spherical symmetry, centered on each atom. We do not adopt this strategy here since our isosurfaces are not smooth at the edge of the molecule and this scheme is numerically more sensitive when calculating the differential conductance maps.

The matching between the electron density calculated from DFT in the proximity of the sample and the evanescent one in the vacuum region is done over an isosurface chosen in such a way that the DFT potential vanishes (it is smaller than a given cutoff). Such an analytic continuation allows us to extract the wave function over the entire region separating the tip and the sample so that the tunneling current can be calculated by using the Bardeen approach. ${ }^{14}$

The current at a given position of the tip $(\vec{r})$ is thus given by

$$
I(V, \vec{r})=4 \pi^{2} \frac{e}{h} \sum_{\mu, \nu}\left[f_{\mu}-f_{\nu}\right]\left|M_{\mu, \nu}(\vec{r})\right|^{2} \delta\left(\varepsilon_{\mu}-\varepsilon_{\nu}-e V\right),
$$

where the sum runs over all the energy levels, $\varepsilon_{i}$, of the tip $(i=\nu)$ and of the sample $(i=\mu), f_{i}$ are the Fermi distributions of both the sample and the tip

$$
f_{i}=\frac{1}{e^{\beta\left(\varepsilon_{i}-E_{F}^{i} \pm V / 2\right)}+1} .
$$

Here $\beta=\frac{1}{K_{B} T}\left(K_{B}\right.$ is the Boltzmann constant and $T$ the temperature), $E_{F}^{i}$ is the Fermi level and $V$ the applied bias. Finally the tip-to-sample tunneling matrix elements, $M_{\mu, \nu}$, are simply obtained by integrating the wave function calculated as described above over a surface $\Sigma$ cutting in between the tip and the sample

$$
\begin{aligned}
M_{\mu, \nu}(\vec{r})= & -\frac{\hbar^{2}}{2 m} \int_{\Sigma}\left[\varphi_{\nu}^{\dagger}\left(\vec{r}-\vec{r}^{\prime}\right) \vec{\nabla} \psi_{\mu}\left(\vec{r}^{\prime}\right)\right. \\
& \left.+-\vec{\nabla} \varphi_{\nu}^{\dagger}\left(\vec{r}-\vec{r}^{\prime}\right) \psi_{\mu}\left(\vec{r}^{\prime}\right)\right] d \vec{S}^{\prime},
\end{aligned}
$$

where $\varphi_{\nu}$ is the tip wave function and $\psi_{\mu}$ is that of the sample. The surface $\Sigma$ is chosen in such a way that the product between the wave function of the tip and that of the substrate assumes a give value.

Our scheme is thus conceptually similar to that of Ref. 20, although our own implementation has been developed independently. ${ }^{21}$ It is more insightful than the more standard Tersoff-Hamann approximation, which does not account for the electronic structure of the tip but still computationally less demanding than fully self-consistent scattering theory. ${ }^{22}$ 


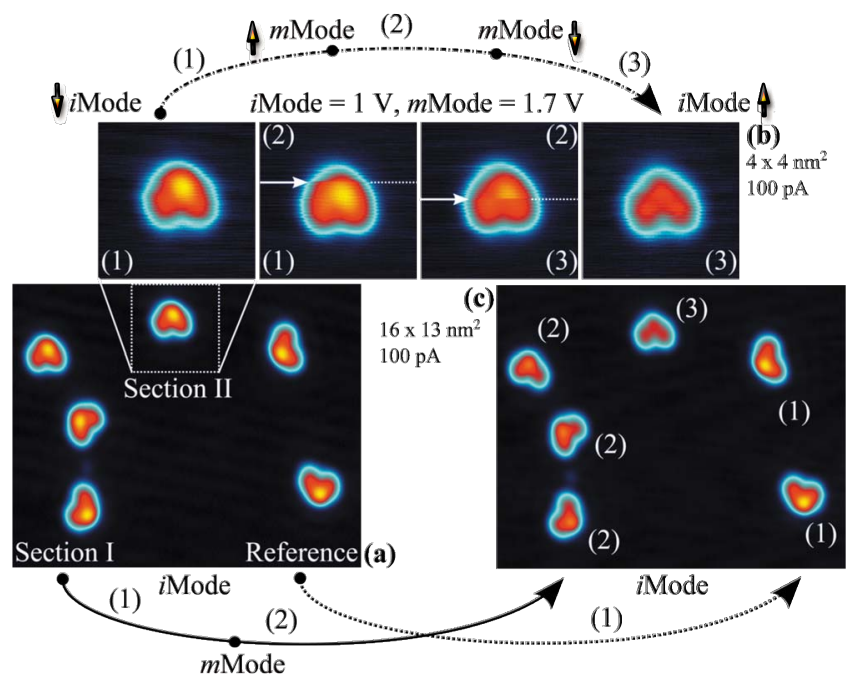

FIG. 2. (Color online) Manipulation of intact Co-Salen (1) by constant-current imaging into its single-(2) and double-(3) dehydrogenated form at a bias voltage above the threshold for manipulation (mMode). (a) An area with six (1) is selected. Two molecules serve as unaltered reference and are only imaged at low bias voltage ( $i$ Mode). Molecules in Sec. I are imaged once, the molecule in Sec. II twice in the $m$ Mode. The top panel (b) shows the same molecule obtained during imaging in the $i$ Mode and the mMode. Whereas $i$ Mode imaging does not affect the molecule, in each of the $m$ Mode images a characteristic jump in the line scans is revealed $(\cdots)$. The first dehydrogenation is detected by a sudden reduction in the molecule apparent height (second panel). During the second dehydrogenation the molecule adopts a mirror symmetric shape (third panel). (c) shows the surface again in the $i$ Mode. The reference molecules remained unmodified, whereas the molecules in Secs. I and II transformed into (2) and (3). The top panel (b) shows the same molecule obtained during imaging in the $i$ Mode and the $m$ Mode (with the scan directions as indicated).

Our STM tip is modeled as a $\mathrm{Cu}$ pyramid oriented along the (111) direction so that it is terminated with a single $\mathrm{Cu}$ atom. The STM currents are calculated initially for different heights ranging between 6 and $10 \AA$, then the topographic STM images are obtained in the constant-current mode. Importantly STS maps are also calculated in the constantcurrent mode, i.e., under the same conditions in which the experiments have been carried out. This eliminates any uncertainty over the interpretation of the $d I / d V$ maps in terms of the molecule's local density of state. ${ }^{23,24}$ Practically we evaluate the tip-to-sample distance $z_{0}(x, y)$ yielding a given current along the molecule $x-y$ plane and then evaluate the STS maps over such a surface.

\section{RESULTS AND DISCUSSION}

Figure 2(a) shows a representative image of six molecules directly after sample preparation as imaged at low bias $(U=1 \mathrm{~V})$. Due to the out-of-plane tilted ethylene bridge, CoSalen is chiral and adsorbs in two enantiomeric shapes in six orientations on the sixfold symmetric surface. ${ }^{10}$ The chirality is manifested by the asymmetric central position.

We will now proceed to demonstrate that sequential dehydrogenation is possible and controllable. Since one of the key tasks is of unequivocally identifying the products of the reaction (i.e., dehydrogenated Co-Salens) we will proceed by analyzing these first.

No data on dehydrogenated Co-Salens are available, so we rely on theory for an unambiguous identification, namely, on the atomistic details as calculated from DFT. The $\mathrm{C}_{2} \mathrm{H}_{4}$ bridge of the $\mathrm{C}_{2} \mathrm{H}_{4} \mathrm{~N}_{2}$ group of the intact molecule (1) adsorbed on the $\mathrm{Cu}(111)$ is tilted by $20^{\circ}$ with respect to the planar position. Thus, the $C_{2}$ symmetry is broken in correspondence of two enantiomeres in the experiment. One hydrogen is pointing toward the $\mathrm{Cu}$ surface [atom 2 of (1), Fig. 1(b)], one pointing out of it [atom 4 of (1), Fig. 1(b)] and the last two remaining approximately in the plane of the molecule. The central Co ion ends up at $2.7 \AA$ above the $\mathrm{Cu}(111)$ hollow site.

The first dehydrogenation removes the most external $\mathrm{H}$, thus leaving (1) with a $\mathrm{C}_{2} \mathrm{H}_{3} \mathrm{~N}_{2}$ apex [(2) of Fig. 1(b)]. In this rearrangement the double hydrogenated $\mathrm{C}$ becomes the most external atom of the molecule and is about $0.3 \AA$ closer to the surface than the most external $\mathrm{C}$ in (1). The Co ion in (2) is displaced by $0.1 \AA$ toward the surface, i.e., the molecule's apparent height has reduced. Notably, also in this case the $C_{2}$ symmetry is broken. The second dehydrogenation [(3), Fig. 1(c)] transforms the Co-Salen apex into a cis-ethyl-diamino $\left(\mathrm{C}_{2} \mathrm{H}_{2} \mathrm{~N}_{2}\right)$ group with a $\mathrm{C}$ double bond and yields a $C_{2}$ symmetry. The two peripheral hydrogens of $\mathrm{C}_{2} \mathrm{H}_{2} \mathrm{~N}_{2}$ lie in the plane of the molecule, which is flat on the surface. This second dehydrogenation brings the entire molecule closer to the surface by $0.3 \AA$ as compared to (1).

Next, we demonstrate our ability to produce the sequential dehydrogenation from (1) by STM manipulation. Imaging at low bias, i.e., $U \leq 1.5 \mathrm{~V}$, leaves the system undisturbed (iMode) and is used to characterize molecules before and after each manipulation cycle. Whereas at elevated bias, i.e., $U>1.5 \mathrm{~V}$, Co-Salens are modified (mMode) and undergo a characteristic and irreversible transition. The transition reflects two dehydrogenations of the $\mathrm{C}_{2} \mathrm{H}_{4}$ bridge equally observed in point manipulation and $m$ Mode imaging with identical intermediate (2) and final (3) products. Figure 2 demonstrates the dehydrogenation during scanning in the $m$ Mode for six neighboring Co-Salens. During the manipulation cycle, two molecules serve as unaltered reference. Once switched (Sec. I), Co-Salen remains chiral whereas the double dehydrogenated Co-Salen (Sec. II) adopts a mirror symmetric appearance at reduced apparent height. Note that the height reduction and the symmetry of the images are already consistent with identifying the products with (2) and (3).

The signature of the dehydrogenation process is also given by two characteristic jumps in the recorded current during local STS experiments as presented in Fig. 3(a). Up to the threshold voltage the current is stable ( $i$ Mode). Beyond that value, i.e., in the $m$ Mode range, sudden current jumps occur regularly. Each change in the current reflects one irreversible dehydrogenation process, highly reproducible with a symmetric final product. The induced dehydrogenation preferentially occurs, when the electron source (tip) is located close to the $\mathrm{C}_{2} \mathrm{H}_{4}$ bridge. As the rate of transitions does not show any nonlinear current dependence, the origin of the processes can be attributed to an energy exchange between a 


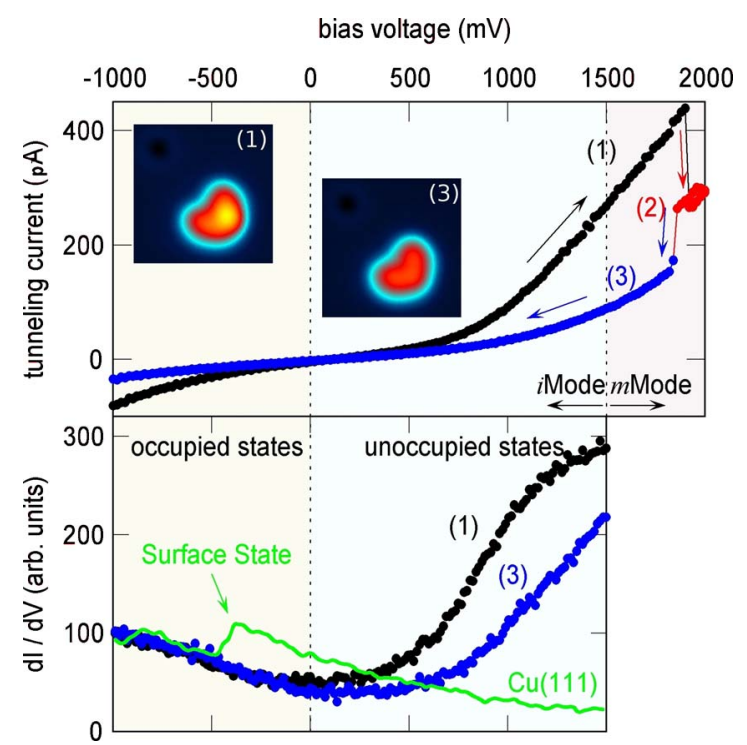

FIG. 3. (Color online) The transformation from (1) into (2) and (3) is efficiently achieved by voltage pulses above the $\mathrm{C}_{2} \mathrm{H}_{4}$ bridge at constant height. With the current monitored, characteristic jumps reveal the moment of dehydrogenation which is only observed above the threshold voltage of $1.5 \mathrm{~V}$. In (a) the recorded current during such a manipulation is presented which shows two abrupt changes. This is indicative for the transition from (1) into (2) and finally (3). Respectively, the images before and after (see insets) show complex (1) and (3). (b) With the voltage limited to $1.5 \mathrm{~V}$, STS data on the differential conductivity manifest a quantitative change in the spectra. Whereas no significant change in the occupied states is found $(U<0)$, unoccupied states $(U>0)$ are shifted toward higher energies after dehydrogenation. The copper spectrum is recorded for the characterization of the tip status at a location nearby with the clear signature of the present surface state.

single tunneling electron $(E=e U>1.5 \mathrm{eV})$ and the ethylene group. Apart from the induced change in molecular appearance no other byproducts are observable in the STM images.

The next and final step consists in confirming with certainty the assignment of the reaction products to the three Co-Salen molecules with different degree of hydrogenation as presented in Fig. 1. The task is particularly challenging since no direct evidence for the atomistic nature of the three molecules observed is available. Our only resource is stateof-the-art electronic-structure theory. Due to the strong electronic coupling of Co-Salen to the metallic substrate, this has to go beyond the mere calculation of the molecular orbitals of the pristine molecule so that our fully quantitative theory of STM both in its topographic and spectroscopical modes is necessary.

Let us begin our analysis by comparing the experimental and the calculated topographs [see Fig. 4(a)]. As one can easily see in the case of both (1) and (2) the largest intensity is always in between the Co center and the $\mathrm{C}_{2} \mathrm{H}_{4} \mathrm{~N}_{2}$ apex and the mirror symmetry is broken. Importantly such a broken symmetry persists for all the applied bias voltages. This is different from what happens for (3), whose topographs remain always symmetric. A more rich analysis can be obtained in the spectroscopical mode.

STS data taken from the sites of the Co ion [Fig. 3(b)] reveal a subtle but significant and characteristic change in the
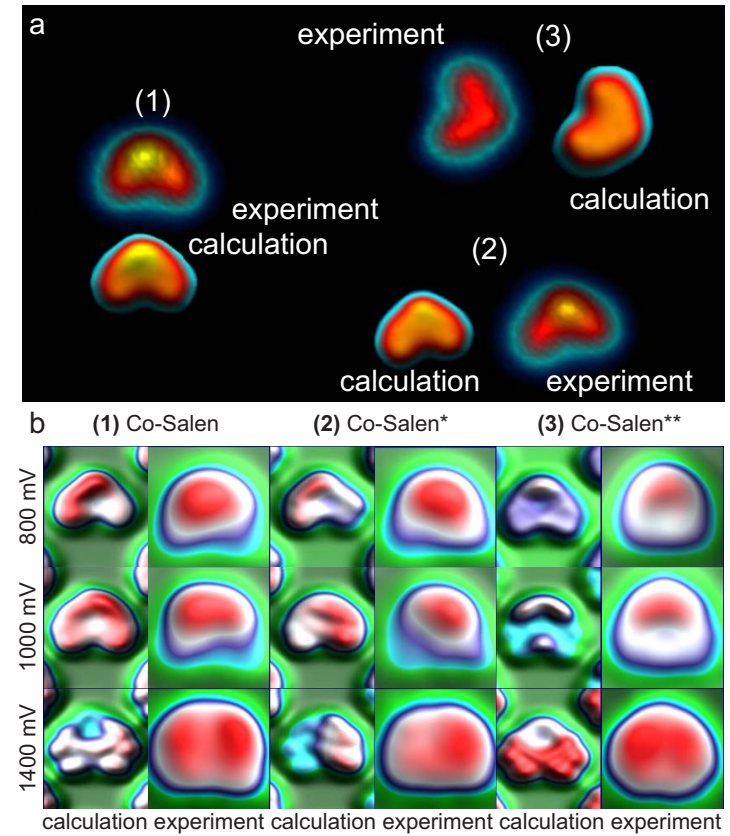

FIG. 4. (Color online) STM/STS maps for the three molecules investigated. In (a) we present the experimental and the simulated topographs of Co-Salen adsorbed on $\mathrm{Cu}(111)$. The images were taken at a voltage of $U=1.4 \mathrm{~V}$. Note that the mirror symmetry is broken for (1) and (2) but not for (3). In panel (b) the experimentally measured STS maps are compared with the calculated ones at three representative voltages of 800,1000 , and $1400 \mathrm{mV}$. The color scale is identical within each row and the very same tip is used for all the images. In the simulated images the periodic replica of the unit cell appear at the edge of the figure. In addition to a general increase in the molecule's lateral extension with bias, we remark three main features: (1) in (1) the largest intensity is on the lefthand side of the $\mathrm{C}_{2} \mathrm{H}_{4} \mathrm{~N}_{2}$ apex and shifts to give a more symmetric image at higher $U ;(2)$ is similar to (1) except that the asymmetry persists at higher $U$; and (3) remains symmetric at all voltages with the maximum intensity at $\mathrm{C}_{2} \mathrm{H}_{2} \mathrm{~N}_{2}$ apex for small $U$ and a shallow minimum at the same position for large $U$.

local density of molecular states before and after the double dehydrogenation. This can be explored in details by STS mapping of the molecular states, i.e., by spatially resolved maps of the differential conductivity at specific energies. The comparison between theory and experiments is presented in Fig. 4(b) where the three molecules are juxtaposed and data are shown for three representative voltages (small $U$ $=800 \mathrm{mV}$, moderate $U=1000 \mathrm{mV}$, and large $U=1400 \mathrm{mV}$ ). The picture demonstrates a substantial quantitative agreement between the experimental and simulated STS maps and allow us to identify with certainty the three different molecules as (1), (2), and (3).

A general feature of the experimental STM/STS maps is the growth of the molecule's lateral extension of the images with bias. This behavior is not completely captured by our simulations as the calculated DFT charge density is continued into the vacuum with a one-dimensional decay function. Therefore, no lateral expansion of the simulated STM images is expected at any bias. Chirality is pronounced through the electron cloud of the sterically out-of-plane repelled hydro- 
gens (of the $\mathrm{C}_{2} \mathrm{H}_{4}$ bridge) for (1) and (2), whereas (3) remains inconspicuous. Going into more details: (1) the STS map for (1) has a pronounced maximum on the left-hand side of the $\mathrm{C}_{2} \mathrm{H}_{4} \mathrm{~N}_{2}$ group at low bias. Toward higher energies, the maximum gradually shifts to the aromatic phenyl ring with a weak asymmetry. These main features are captured well by the theory. (2) (2) appears similar to (1) with a more pronounced asymmetry, in particular, at high bias. At high bias the contrast is dominated by half of the molecule, again in good agreement with the simulated images. (3) (3) maintains the median plane symmetry at all voltages. The most relevant feature is the extended intensity maximum at low and moderate biases $(0.5$ and $0.8 \mathrm{~V})$ located at the $\mathrm{C}_{2} \mathrm{H}_{2} \mathrm{~N}_{2}$ apex and an intensity depression just below that. At high bias the maximum intensity extends over the entire molecule and a moderate depression develops at the $\mathrm{C}_{2} \mathrm{H}_{2} \mathrm{~N}_{2}$ apex. Also in this case the relevant features are reproduced by the simulated maps.

Having identified with certainty the reaction products we now turn to the energy needed for the dehydrogenation, i.e., the energy associated with the following two reactions:

$$
\begin{aligned}
\mathrm{C}_{2} \mathrm{H}_{4} \mathrm{~N}_{2} & \rightarrow \mathrm{C}_{2} \mathrm{H}_{3} \mathrm{~N}_{2}+\frac{1}{2} \mathrm{H}_{2}, \\
\mathrm{C}_{2} \mathrm{H}_{4} \mathrm{~N}_{2} & \rightarrow \mathrm{C}_{2} \mathrm{H}_{2} \mathrm{~N}_{2}+\mathrm{H}_{2} .
\end{aligned}
$$

These are evaluated from the difference in total energy between the intact molecule and the two products of reaction as calculated by DFT. The reference energy for hydrogen is that of the $\mathrm{H}_{2}$ molecule in vacuum. We obtain $1.49 \mathrm{eV}$ and 1.75 $\mathrm{eV}$, respectively, for the single and double dehydrogenation. These calculated values are thus in rather good agreement with the voltages necessary for dehydrogenation, which are larger than $1.5 \mathrm{~V}$.

Before concluding we wish to relate the STM data to the electronic structure of Co-Salen. In Fig. 5 we present the electronic wave function isosurface of the Co-Salen molecular orbitals comprised in the $(-0.5,1.5) \mathrm{eV}$ energy window, i.e., of those molecular orbitals responsible for the STM current. The molecule is considered in vacuum and for simplicity we limit our analysis to (1) and (3) (Fig. 5). In general the two molecules present a rather similar energy-level structure and, in particular, the first three highest occupied molecular orbitals (HOMOs, $E<0)$ present little differences in the two cases. Thus, one expects that the STM features of the occupied states, explored at negative voltage, depend little on the amount of $\mathrm{H}$ at the $\mathrm{C}_{2} \mathrm{H}_{4} \mathrm{~N}_{2}$ apex. Such an expectation is indeed confirmed by the differential conductance $d I / d U$ obtained from single point spectroscopy [see Fig. 3(b)]. STS data at negative bias voltage are fairly independent of the type of molecule probed and tells little about the $\mathrm{H}$ content of Co-Salen.

The situation is different for the lowest occupied molecular orbitals (LUMOs). In fact, while all the LUMOs of the double dehydrogenated molecule (3) are symmetric with respect to the nodal plane passing through the Co, those of the intact molecule (1) displays an evident symmetry breaking. For this reason one expects that both the topography and the spectroscopical maps taken at positive voltage (exploring

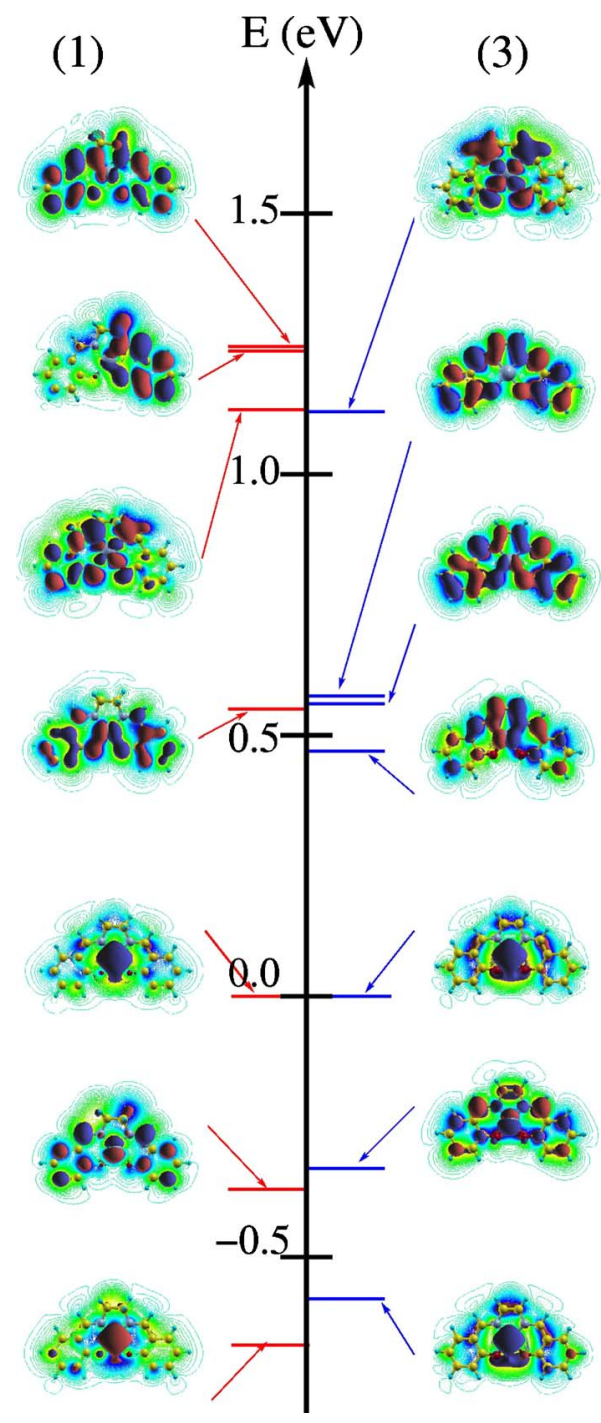

FIG. 5. (Color online) Electronic wave-function isosurfaces of the frontier molecular orbitals of Co-Salen in the (1) (left) and (3) (right) configurations. The different colors represent the sign of the wave function and the levels are align to have the HOMO at $E=0$. Note that, while the filled states are all symmetric with respect to a median plane passing through the Co, the empty ones of molecule (1) are asymmetric. Note also that the asymmetry develops for level at energies larger than $1 \mathrm{eV}$ above the HOMO while the STM images are asymmetric already for voltages of around $0.5 \mathrm{~V}$.

empty states) remain symmetric for (3) but display asymmetry for (1), as extensively proved by the STM data.

Note, however, that the spatial distribution of the wave function for the LUMOs does not match directly the experimental spectroscopical maps, i.e., the knowledge of the local density of states for the free molecule alone is not helpful in describing the STM images. For instance for (1) one expects asymmetry originating from the electronic structure only for scans at voltages larger than about $1 \mathrm{~V}$, i.e., at the voltage at which the first asymmetric LUMO starts to contribute to the current. In contrast STM data reveals asymmetry even at lower bias $(\sim 0.5 \mathrm{~V})$, although certainly this is enhanced as the bias is increased. Our rigorous STM simulations for the molecule on the substrate, which include both the molecule- 
substrate interaction and the corrections due to the molecule's local height, obviate such a problem and allow a direct comparison between theory and experiments. This is a crucial aspect of the present work.

In any case the main electronic differences between (3) and both (1) and (2) can be easily rationalized by considering the nature of the $\mathrm{C}-\mathrm{C}$ bond of the $\mathrm{C}_{2} \mathrm{H}_{4} \mathrm{~N}_{2}$ apex. In fact both (1) and (2) are characterized by a single C-C bond, which effectively breaks the extended $\pi$ conjugation of the molecule. The double dehydrogenation leading to (3) transforms such a single bond into a double one. As a consequence an extended $\pi$ orbital now spreads over the entire molecule and dominates both occupied and empty states, with the exception of the HOMO, which is formed entirely by a Co $d$ level. Such an extended $\pi$ orbital is characterized by a rather uniform charge distribution over the molecule and a strong interaction with the substrate. Therefore the molecule appears lower on the substrate and without any distinct atomic feature.

\section{CONCLUSION}

In conclusion we have investigated the ultimate limit offered by STM techniques for both imaging small molecules and manipulating their geometry. In particular, we have dem- onstrated that sequential dehydrogenation of the planar CoSalen is possible with both single point spectroscopy and slow scan at high voltage. In the process, we have been able to detect not only the final product of the dehydrogenation but also an intermediate reaction product in which a single $\mathrm{H}$ atom is dissociated. This is a particularly delicate test for STM since the reaction products do not show any specific change in bonding configuration and they are not known $a$ priori. Thus the interpretation of the data is done by comparing the experimental data with state-of-the-art STM simulations based on density-functional theory. This work demonstrates that STM combined with theory can be a fully quantitative tool for studying surface chemistry.

\section{ACKNOWLEDGMENTS}

This work is funded by the SpiDME European project (Sixth Framework Program, NEST). S.S. and N.B. acknowledge CRANN and Science Foundation of Ireland (08/ERA/ 11759) for additional financial support. S.K., J.B., G.H., and R.W. acknowledge additional funding through the GrK 611 and the SFB 668 TP A4 of the Deutsche Forschungsgemeinschaft and the Cluster of Excellence NANOSPINTRONICS. Computational resources have been provided by the HEA IITAC project managed by the Trinity Center for High Performance Computing and by ICHEC.
${ }^{1}$ T. Komeda, Y. Kim, M. Kawai, B. N. J. Persson, and H. Ueba, Science 295, 2055 (2002).

${ }^{2}$ J. I. Pascual, N. Lorente, Z. Song, H. Conrad, and H.-P. Rust, Nature (London) 423, 525 (2003).

${ }^{3}$ B. C. Stipe, M. A. Rezaei, W. Ho, S. Gao, M. Persson, and B. I. Lundqvist, Phys. Rev. Lett. 78, 4410 (1997).

${ }^{4}$ P. A. Sloan and R. E. Palmer, Nature (London) 434, 367 (2005).

${ }^{5}$ S.-W. Hla, L. Bartels, G. Meyer, and K.-H. Rieder, Phys. Rev. Lett. 85, 2777 (2000).

${ }^{6}$ Y. Kim, T. Komeda, and M. Kawai, Phys. Rev. Lett. 89, 126104 (2002).

${ }^{7}$ L. J. Lauhon and W. Ho, J. Phys. Chem. A 104, 2463 (2000).

${ }^{8}$ H. Lesnard, M.-L. Bocquet, and N. Lorente, J. Am. Chem. Soc. 129, 4298 (2007).

${ }^{9}$ P. G. Cozzi, Chem. Soc. Rev. 33, 410 (2004).

${ }^{10}$ S. Kuck, S.-H. Chang, J.-P. Klöckner, M. H. Prosenc, G. Hoffmann, and R. Wiesendanger, ChemPhysChem 10, 2008 (2009).

${ }^{11}$ J. A. A. W. Elemans, S. J. Wezenberg, M. J. J. Coenen, E. C. Escudero-Adán, J. Benet-Buchholz, D. den Boer, S. Speller, A. W. Kleij, and S. De Feyter, Chem. Commun. 46, 2548 (2010).

${ }^{12}$ S. Kuck, J. Wienhausen, G. Hoffmann, and R. Wiesendanger,
Rev. Sci. Instrum. 79, 083903 (2008).

${ }^{13}$ C. Wittneven, R. Dombrowski, S.-H. Pan, and R. Wiesendanger, Rev. Sci. Instrum. 68, 3806 (1997).

${ }^{14}$ J. Bardeen, Phys. Rev. Lett. 6, 57 (1961).

${ }^{15}$ J. M. Soler, E. Artacho, J. D. Gale, A. Garcia, J. Junquera, P. Ordejon, and D. Sanchez-Portal, J. Phys.: Condens. Matter 14, 2745 (2002).

${ }^{16}$ N. Troullier and J. L. Martins, Phys. Rev. B 43, 8861 (1991).

${ }^{17}$ J. Zhu, X. W. Wang, and S. G. Louie, Phys. Rev. B 45, 8887 (1992).

${ }^{18}$ J. P. Perdew, K. Burke, and M. Ernzerhof, Phys. Rev. Lett. 77, 3865 (1996).

${ }^{19}$ J. Tersoff and D. R. Hamann, Phys. Rev. Lett. 50, 1998 (1983).

${ }^{20}$ O. Paz and J. M. Soler, Phys. Status Solidi B 243, 1080 (2006).

${ }^{21}$ N. Baadji and S. Sanvito (unpublished).

${ }^{22}$ C. Toher, I. Rungger, and S. Sanvito, Phys. Rev. B 79, 205427 (2009).

${ }^{23}$ W.-H. Soe, C. Manzano, A. De Sarkar, N. Chandrasekhar, and C. Joachim, Phys. Rev. Lett. 102, 176102 (2009).

${ }^{24}$ M. Ziegler, N. Néel, A. Sperl, J. Kröger, and R. Berndt, Phys. Rev. B 80, 125402 (2009). 